\title{
Numerical simulations of vertical oscillations of a multi-stranded coronal loop
}

\author{
M. Gruszecki ${ }^{1}$, K. Murawski ${ }^{1}$, M. Selwa ${ }^{1}$, and L. Ofman ${ }^{2}$ \\ ${ }^{1}$ Group of Astrophysics and Gravity Theory, Institute of Physics, UMCS, ul. Radziszewskiego 10, 20-031 Lublin, Poland \\ e-mail:marcingruszecki@wp.pl \\ 2 The Catholic University of America, NASA Goddard Space Flight Center, Code 612.1, Greenbelt, MD 20771, USA
}

Received 12 April 2006 / Accepted 5 September 2006

ABSTRACT

\begin{abstract}
Aims. We consider impulsively generated oscillations in a 2D model of a curved solar coronal arcade loop that consists of up to 5 strands of dense plasma.

Methods. First we do a simulation for a loop which consists of two curved strands. We evaluate by means of numerical simulations the influence of the distance between the strands and their number on wave period, attenuation time, and amplitudes of standing kink waves.

Results. The results of the numerical simulations reveal that only strands which are very close to each other (distance comparable to the strand width) considerably change the collective behavior of kink oscillations. More distant strands also exhibit weak coupling of the oscillations. However, their behavior can essentially be explained in terms of separate oscillating loops. We compare the numerical results with recent TRACE observational findings, and find qualitative agreement.
\end{abstract}

Key words. magnetohydrodynamics (MHD) - Sun: corona - methods: numerical

\section{Introduction}

Magnetic loops are the main building blocks of solar corona, that are able to sustain various kinds of waves and oscillations. The waves may play an important role in the heating of the loops, as was first suggested by Ionson (1978), and also can be used as a diagnostic tool of the loop parameters (e.g., Nakariakov \& Ofman 2001).

Wave modes relevant for coronal loops were studied analytically few decades ago by Edwin \& Roberts (1982) and Roberts et al. (1984) who in their seminal papers adopted slab and cylindrical geometries of a straight magnetic structure. Recently these geometries were generalized to curved magnetic field topologies (e.g., Brady \& Arber 2005; Del Zanna et al. 2005; Murawski et al. 2005a,b; Brady et al. 2006; Diáz et al. 2006; Verwichte et al. 2006a-c; Selwa et al. 2005, 2006a,b). Among different modes a particular interest was focused on fast magnetosonic standing kink waves (e.g., Nakariakov \& Verwichte 2005), which were recently simulated numerically, among others, by Brady \& Arber (2005), Brady et al. (2006), and Selwa et al. (2006a). As far as polarization is concerned there are two known modes of standing kink waves: transverse oscillations which occur in a plane perpendicular to the loop plane and vertical oscillations which are transverse oscillations in the plane of the loop (Nakariakov \& Verwichte 2005; Diáz et al. 2006). First direct observations of kink waves in coronal loops in Extreme Ultraviolet emission lines (EUV) by the TRACE satellite (Handy et al. 1998) were reported by Aschwanden et al. (1999), Nakariakov et al. (1999), Schrijver et al. (2002), Aschwanden et al. (2002), and Verwichte et al. (2004). The first observational evidence of vertical oscillations of solar coronal loops was provided by Wang \& Solanki (2004) who noted the periodic, decaying expansion and contraction of an active-region loop that was recorded by TRACE. They reported an initial velocity of the loop of $\sim 130 \mathrm{~km} \mathrm{~s}^{-1}$ and a displacement amplitude of the loop summit of $7900 \mathrm{~km}$. The loop expanded and shrank with an oscillation period of $\sim 3.9$ min. These oscillations were quickly damped with a decay time of $11.9 \mathrm{~min}$. The loop length was estimated as $300-400 \mathrm{Mm}$.

These observational findings were confirmed by the results of recent numerical studies (Brady \& Arber 2005; Murawski et al. 2005a,b; Selwa et al. 2005; Brady et al. 2006; Diáz et al. 2006; Verwichte et al. 2006a-c) in which the monolithic loop model was adopted. However recently, Aschwanden (2005) concluded from the TRACE data that the loop observed by EIT/SOHO (Schmelz et al. 2003) is highly structured as it consists of at least 10 strands of width less than $2 \mathrm{Mm}$ each (Aschwanden \& Nightingale 2005).

Early work on waves in complex loop structures was done by Murawski (1993) who by means of numerical simulations considered a simple structure of two-parallel slabs. He showed that an energy leakage from these slabs, associated with the propagation of sausage and kink fast magnetosonic waves, is responsible for a cross-talk, or coupling between the slabs.

Recently, Ofman (2005) investigated the oscillation of multithreaded loops by solving resistive 3D MHD equations to model straight cylindrical multi-stranded loop. Ofman (2005) found that the coupling between closely spaced strands affects the damping rate of the oscillations, which becomes more strongly dependent on the value of the Lundquist number, compared to the monolithic loop. However, in the present study we neglect the effect of resistivity and concentrate on the ideal damping of fast mode oscillations in multi-stranded curved loop arcade.

In this paper we refer to the observationally determined signatures of the vertical oscillations (Wang \& Solanki 2004). We realize our goal by taking into account a simple two-dimensional 
geometry of a coronal loop arcade in which loop oscillations are excited by initially launched localized pulses. In the present paper we extend the models developed by Brady \& Arber (2005); Del Zanna et al. (2005); Murawski et al. (2005a,b); Brady et al. (2006); Diáz et al. (2006); Verwichte et al. (2006a-c), and Selwa et al. $(2005,2006 a, b)$ to a loop that consists of up to five strands.

This paper is organized as follows: the next section presents the details of the numerical models. Section 3 contains numerically obtained results. This paper is concluded by a summary of the main results.

\section{Numerical models}

We perform numerical simulations in a two-dimensional magnetically structured atmosphere. We neglect gravity and nonideal effects. As a consequence, we use the ideal magnetohydrodynamic (MHD) equations to describe the coronal plasma:

$\frac{\partial \varrho}{\partial t}+\nabla \cdot(\varrho \boldsymbol{V})=0$,

$\varrho \frac{\partial \boldsymbol{V}}{\partial t}+\varrho(\boldsymbol{V} \cdot \nabla) \boldsymbol{V}=-\nabla p+\frac{1}{\mu}(\nabla \times \boldsymbol{B}) \times \boldsymbol{B}$,

$\frac{\partial \boldsymbol{B}}{\partial t}=\nabla \times(\boldsymbol{V} \times \boldsymbol{B})$,

$\frac{\partial E}{\partial t}+\nabla \cdot\left[\left(E+p_{\mathrm{T}}\right) \boldsymbol{V}-\frac{\boldsymbol{B}(\boldsymbol{B} \cdot \boldsymbol{V})}{\mu}\right]=0$.

Here $\varrho$ is mass density, $\boldsymbol{V}$ is flow velocity, $p$ is gas pressure, $\boldsymbol{B}$ is magnetic field $(\nabla \cdot \boldsymbol{B}=0)$ and $\mu$ is the magnetic permeability. The symbol $p_{\mathrm{T}}$ denotes the total pressure which represents a sum of $p$ and magnetic pressure $B^{2} /(2 \mu)$, viz.

$p_{\mathrm{T}}=p+\frac{B^{2}}{2 \mu}$

Plasma energy density $E$ can be expressed as a sum of kinetic, internal, and magnetic energy densities:

$E=\frac{\varrho V^{2}}{2}+\frac{p}{\gamma-1}+\frac{B^{2}}{2 \mu}$.

Here $\gamma$ is the adiabatic index which for the ideal plasma is equal to $5 / 3$. We assume that plasma quantities are independent of the spatial coordinate $y$ and set $V_{y}=B_{y}=0$. As a consequence of this assumption the Alfvén wave is removed from the system, leaving only the fast and slow magnetosonic waves.

\subsection{Initial configuration}

In this section we present a model of a curved coronal loop that consists of up to five strands. All equilibrium quantities are denoted by subscript e. We adopt and modify the model which was originally described by Oliver et al. (1993) and recently used by Murawski et al. (2005b) and Selwa et al. (2005, 2006a,b). A modification of this model is based on the introduction of smooth strands. As a result, a curved arcade loop consists of separate strands of condensed plasma.

We assume that at the equilibrium there is no pressure gradient, $\nabla p_{\mathrm{e}}=0$. As a consequence the force balance condition is:

$\left(\nabla \times \boldsymbol{B}_{\mathrm{e}}\right) \times \boldsymbol{B}_{\mathrm{e}}=0$.
This condition can be satisfied by the current-free magnetic field

$\nabla \times \boldsymbol{B}_{\mathrm{e}}=0$.

Equation (8) is solved with the use of the magnetic potential $A$, viz.

$\boldsymbol{B}_{\mathrm{e}}=\nabla \times(A \hat{\boldsymbol{y}})$.

Here $\hat{\boldsymbol{y}}$ is a unit vector along the $y$-direction and a magnetic potential is given as

$A(x, z)=B_{0} \Lambda_{\mathrm{B}} \cos \left(x / \Lambda_{\mathrm{B}}\right) \mathrm{e}^{-z / \Lambda_{\mathrm{B}}}$.

As a consequence, the equilibrium magnetic field components are

$B_{\text {ex }}=B_{0} \cos \left(x / \Lambda_{\mathrm{B}}\right) \mathrm{e}^{-z / \Lambda_{\mathrm{B}}}$,

$B_{\mathrm{ez}}=-B_{0} \sin \left(x / \Lambda_{\mathrm{B}}\right) \mathrm{e}^{-z / \Lambda_{\mathrm{B}}}$.

Here $B_{0}$ is the magnetic field at the reference level $z=0$ and the magnetic scale height is

$\Lambda_{B}=\frac{2 L}{\pi}$

where $L$ is the horizontal half-width of the arcade, chosen as $L=$ $100 \mathrm{Mm}$. We assume now that at the equilibrium mass density is constant, $\varrho_{\mathrm{e}}=$ const. For the above choice of the magnetic field the Alfvén speed $V_{\mathrm{Ae}}=\left|B_{\mathrm{e}}\right| / \sqrt{\mu \varrho_{\mathrm{e}}}$ decays exponentially with height $z$ but the decay rate is small. Constant background pressure, $p_{\mathrm{e}}=\beta B_{0}^{2} /(2 \mu)=$ const., and constant mass density, $\varrho_{\mathrm{e}}=$ const., implies that background temperature $T_{\mathrm{e}} \sim p_{\mathrm{e}} / \varrho_{\mathrm{e}}=$ const. Here $\beta=p_{\mathrm{e}} /\left(B_{\mathrm{e}}^{2} / 2 \mu\right)$ is the plasma beta which for the chosen set of equilibrium parameters is equal to 0.012 at $z=0$.

Strands are modeled by the following mass density profile:

$\varrho(x, z)=\frac{\varrho_{\mathrm{e}}}{2} \sum_{k=1}^{n}\left|\operatorname{erf}\left(\frac{A-A_{k-1}}{\sigma}\right)-\operatorname{erf}\left(\frac{A-A_{k}}{\sigma}\right)\right|$,

where $A_{k-1}=A\left(L_{\mathrm{f}}-a_{k-1}, 0\right)<A_{k}=A\left(L_{\mathrm{f}}-a_{k}, 0\right)$, erf is erf function and $\sigma$ denotes a sharpness of strands edges. We choose and hold fixed $\sigma=\sqrt{0.05}(A), L_{\mathrm{f}}=0.7 L, a=0.0125 L$, while $a_{n}, n \geq 2$ are allowed to vary. For such choice of parameters lengths of the inner strands are not fixed and they vary with $d$ which denotes the distance between two neighbor strands, evaluated at their summits. These strands are denser than the ambient corona, $\varrho_{\mathrm{i}}=11 \varrho_{\mathrm{e}}$, where $\varrho_{\mathrm{e}}$ is mass density in the ambient medium while $\varrho_{\mathrm{i}}$ is mass density within the strands, which are not of ideal circular shape. In a case of two strands the average radius and length of the outer strand are equal to $\sim 70 \mathrm{Mm}$ and $\sim 190 \mathrm{Mm}$, respectively (Fig. 1). As a consequence of the constant background pressure and mass density enhancements, these strands correspond to regions of cold, condensed plasma.

It is noteworthy that the Alfvén speed within the strands is $\sqrt{\varrho_{\mathrm{i}} / \varrho_{\mathrm{e}}}=\sqrt{11}$ times smaller than in the ambient medium. As a result, these strands are cavities for fast magnetoacoustic waves.

\subsubsection{Perturbations}

As we are interested in impulsively excited waves we launch an initial pulse in gas pressure, i.e.

$p(x, z, t=0)=p_{\mathrm{e}}\left\{1+A_{\mathrm{p}} \mathrm{e}^{-\left(x^{2}+z^{2}\right) / w^{2}}\right\}$.

Here $w$ is the initial pulse width. In the simulations we choose the relative pulse amplitude $A_{\mathrm{p}}=15$ and width $w=0.35 \mathrm{~L}$. 


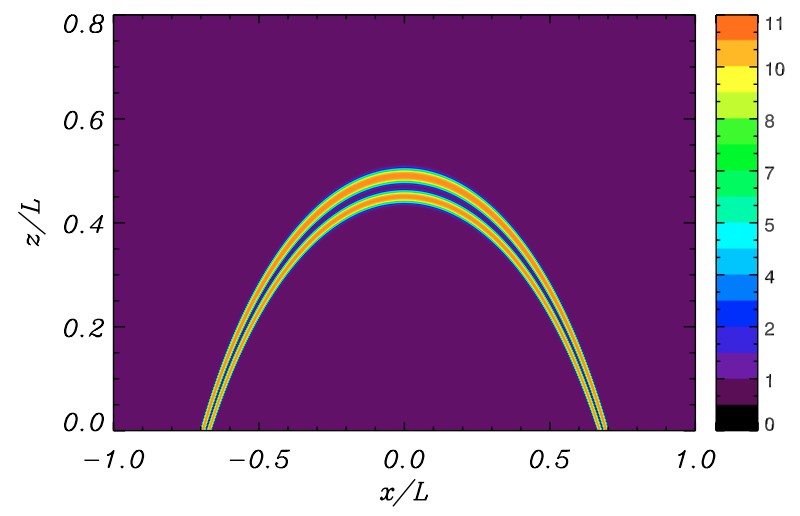

Fig. 1. Equilibrium mass density profiles representing two strands of a coronal loop.

\section{Numerical results}

Numerical results are obtained with the use of the code EMILY (Jones et al. 1997). This code solves the initial-value problem for the time-dependent non-ideal magnetohydrodynamic equations, using an explicit-implicit algorithm. The algorithm is a finitevolume scheme that implements an approximate Riemann solver for the hyperbolic fluxes. As a result, the implemented numerical scheme is second-order accurate in space and time.

We set two-dimensional Eulerian box $(-L, L) \times(0,2 L)$ which is covered by $600 \times 800$ numerical cells. This grid is sufficient to resolve the required spatial scales that are not much affected by numerical diffusion. We set open boundary conditions at the left, right and top of the simulation region. We apply line-tying boundary condition at the remaining boundary. This condition is sustained by setting all velocity components to zero $\boldsymbol{V}=0$, while for the remaining plasma quantities zero-gradient boundary conditions are established. As a result of an implementation of the line-tying boundary condition magnetic field lines are frozen to their initial locations at the boundaries. Such choice of the boundary conditions results in a wave signal leaving freely the simulation region where the open boundary conditions are implemented while a wave signal is reflected from the boundary where the line-tying boundary condition is implemented.

As in the simulations we used a code which is based on the method of characteristics, ideally, no reflection occurs from open boundaries. However, in practice, there is some small amount of reflecting signal. We have verified by moving the top boundary farther away that the boundary set at $z=2 L$ is sufficiently far away from the loop's apex that no numerical artifacts were influencing the results of this study.

\subsection{A double-stranded loop}

In this section we present and discuss numerical results on oscillations of a double-stranded curved loop. It is noteworthy that both strands have widths that can be resolved with TRACE in terms of density inhomogeneity over the crosssection (Aschwanden 2005).

In our parametric study we vary the distance between the two strands, $d$, and look for the influence on wave characteristics. This distance is varied within the interval $0-15 \mathrm{Mm}$, inferred from the EIT observations which reveal that the mean loop width is $19 \pm 7 \mathrm{Mm}$ (Schmelz et al. 2003).

Both strands start to oscillate when fast magnetoacoustic waves, triggered by the initial pulse, reach their summits.

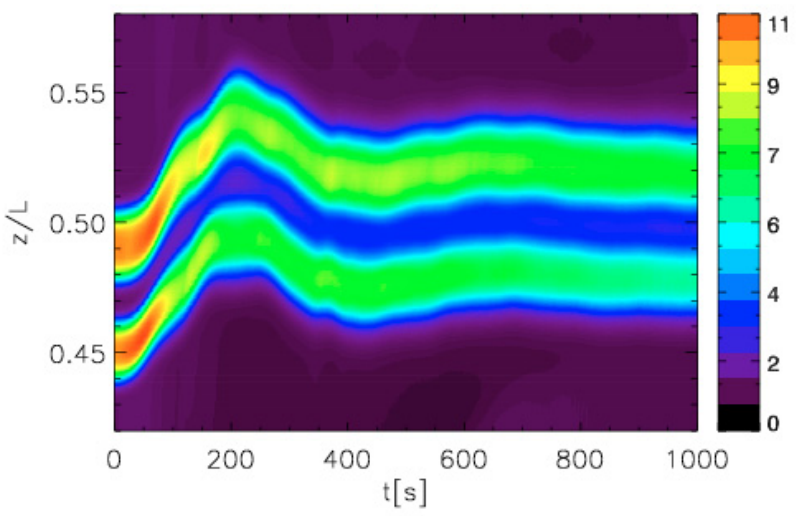

Fig. 2. Time-signatures of the mass density (colour scale; arbitrary units), collected at the strands summits. A distance between the summits of two strands is $d=4 \mathrm{Mm}$. Spatial coordinate $z$ and time $t$ are measured in units of $\mathrm{L}$ and in seconds, respectively.

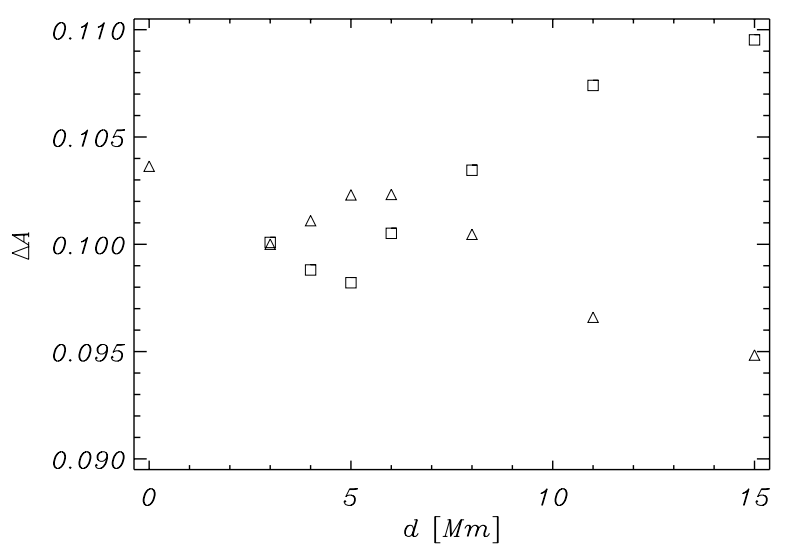

Fig. 3. Relative wave amplitude $\Delta A$ for the outer (triangles) and inner (squares) strands vs. the distance between the summits of two strands $d$. $\Delta A$ and $d$ are measured in units of the corresponding apex height and in $\mathrm{Mm}$, respectively.

Figure 2 displays time-signatures of the mass density, evaluated by collecting the signal at the strands apices. It is discernible that strands oscillate. But these oscillations decay with attenuation time $\tau$. The strands do not return to their initial positions, displaying offsets. Similar offsets were observed and explained by Selwa et al. $(2005,2006 a)$ in their numerically obtained data for a monolithic loop.

Figure 3 shows relative wave amplitude $\Delta A$ vs. $d$ for the outer (triangles) and inner (squares) strands. Relative wave amplitude is normalized by the height of the corresponding apex. For example in Fig. $2 \Delta A$ is evaluated at $t \simeq 200 \mathrm{~s}$ when the outer strand is shifted outwards to $A(t \simeq 200 \mathrm{~s}) \simeq 54.05 \mathrm{Mm}$. As the apex height is $h \simeq 49.09 \mathrm{Mm}(h=A(t=0 \mathrm{~s}))$ we obtain $\Delta A=[A(t \simeq 200 \mathrm{~s})-A(t=0 \mathrm{~s})] / h \simeq 0.101$. For $d \in(5 ; 15) \mathrm{Mm}$ we observe that $\Delta A$ for the outer strand decreases with $d$, while $\Delta A$ increases with $d$ for the inner strand. For a larger value of $d$, the lower strand acquires more energy as it is closer to the initial pulse and its relative oscillation amplitude attains a higher level. Simultaneously, the amplitude of the outer strand's oscillations decreases, as more energy of the initial pulse was trapped by the lower strand. As a consequence of a smaller value of $\Delta A$ outer 

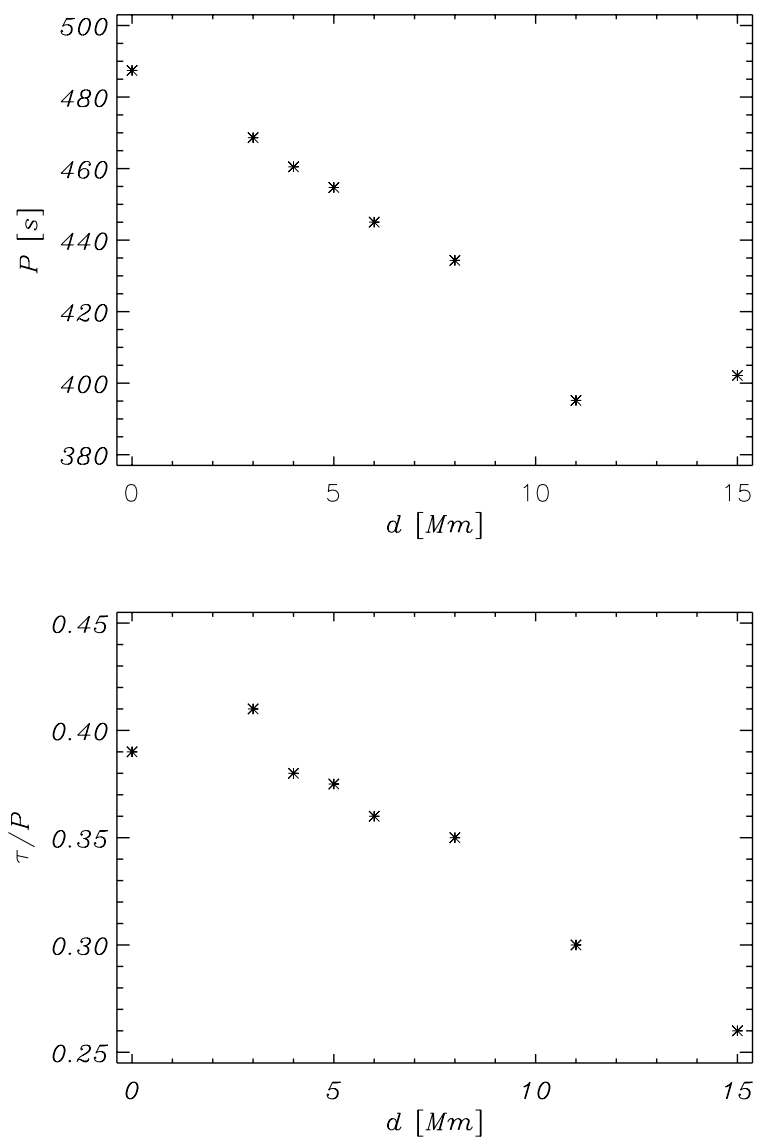

Fig. 4. Wave period $P$ (top panel) and the ratio of the attenuation time to the wave period, $\tau / P$, (bottom panel), evaluated in the outer strand vs. a distance between the summits of two strands, $d$.

strand is stretched less, its length is smaller, and the period of vertical oscillations decreases with $d$ (Fig. 4, top panel).

We conclude from Fig. 3 that for the range of $d \in(3 ; 5) \mathrm{Mm}$ $\Delta A$ evaluated for the inner and outer strands exhibit opposite trends, but the period $P$ trend is the same in both cases (Fig. 4, top panel). The only explanation of such scenario results from the fact that the strands lying within the range of the double width of the strand exhibit a cross-talk (Murawski 1993), while for $d>5 \mathrm{Mm}$ the strands act essentially like separate parts of a two-stranded loop. Indeed, Fig. 5 shows cross-sections of mass density profiles for the outer and inner strands, shown at three different times. At $t=0$ we see an initial configuration which corresponds to the strands which are separated by $d=4 \mathrm{Mm}$. At $t=30 \mathrm{~s}$ waves which originate from the initial pulse hits the inner strand and as a result this strand is compressed. At $t=60 \mathrm{~s}$ it is clear that waves reach the outer strand and cause an increase of its mass density. Simultaneously the inner strand becomes rarified. At $t=90 \mathrm{~s}$ it is discernible that the strands partially overlap and start to oscillate essentially as one wide loop.

We conclude that the magnitudes of $\Delta A$ for the inner and outer strands get closer as the strands are located closer to each other (Fig. 3). For $d=3 \mathrm{Mm}$ both strands have very similar lengths and they are located essentially at similar distances from the initial pulse. As a consequence, $\Delta A$ attains the same value for the outer and inner strands. For $d \in(0 ; 3) \mathrm{Mm}$ after $t \simeq 200 \mathrm{~s}$ the strands partially overlap each other and they oscillate essentially as a single entity.

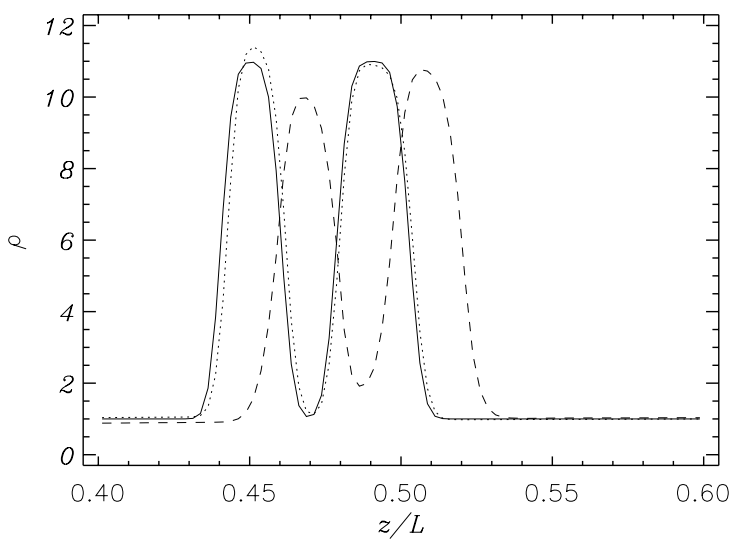

Fig. 5. Cross sections of mass density profiles for outer and inner strands along $x / L=0$ and $z / L \in(0.4 ; 0.6)$ : at $t=0$ (solid line), $t=30 \mathrm{~s}$ (dotted line) and $t=90 \mathrm{~s}$ (dashed line). A distance between two strands is $d=4 \mathrm{Mm}$.

We evaluate the wave period $P$ and the ratio of the attenuation time $\tau$ to wave period $P, \tau / P$, for the outer strand. Values of $P$ and $\tau$ are obtained by fitting time-signatures of simulated vertical location of the loop's apex vs. time to an attenuated time signature (Selwa et al. 2005).

Brady \& Arber (2005), Brady et al. (2006), Diáz et al. (2006), Verwichte et al. (2006a-c) and Selwa et al. (2006a,b) showed that energy leakage can be the mechanism that is responsible for the attenuation of vertical kink oscillations. From Fig. 2 we conclude that for a multi-stranded loop such a mechanism is indeed efficient as it leads to rapidly decaying waves. We note that $\tau / P$ declines with $d$, attaining a value of 0.39 for $d=0 \mathrm{Mm}$ and 0.26 for $d=13 \mathrm{Mm}$ (Fig. 4). As a consequence, we infer that the distance between the strands plays a significant role in wave attenuation.

It is worth mentioning the case of $d=0$. For this distance both strands cover each other and there is one strand but with the mass density doubled. From Fig. 4 it is clear that values of $P$ and $\tau / P$ for such twice denser strand are highest. As a consequence, a denser loop traps more energy and it oscillates with a higher period. These results are in an agreement with Smith et al. (1997) who showed that energy leakage is smaller for a denser loop. The fact that $\tau / P$ grows with the density ratio was confirmed recently by Verwichte et al. (2006a), Diáz et al. (2006), and Selwa et al. (2006b).

\subsection{A multi-stranded loop}

In this section we present numerical results on oscillations of the multi-stranded loop that consists of up to five strands. These strands are separated by $d=4 \mathrm{Mm}$ and $d=10 \mathrm{Mm}$. In our parametric study we vary the number of strands $n$, and look for its influence on wave characteristics. We start with the dependance of $\Delta A$ on $n$. Figure 6 shows that $\Delta A$ grows (declines) with $n$ for $d=4 \mathrm{Mm}(d=10 \mathrm{Mm})$. This peculiar behavior results from a coherent interaction between the strands for $d=4 \mathrm{Mm}$ and essentially a lack of interaction for $d=10 \mathrm{Mm}$. As $\Delta A$ becomes larger for a larger value of $n$ (Fig. 6, top panel), the offset attains a higher value and the loop becomes longer. As a consequence of that, such longer loop exhibits longer period oscillation. Indeed, Fig. 7 (top panel) shows that $P$ varies from $410 \mathrm{~s}$ for $n=1$ up to $530 \mathrm{~s}$ for $n=5$. Due to the increase of $P$ with $n, \tau / P$ declines 

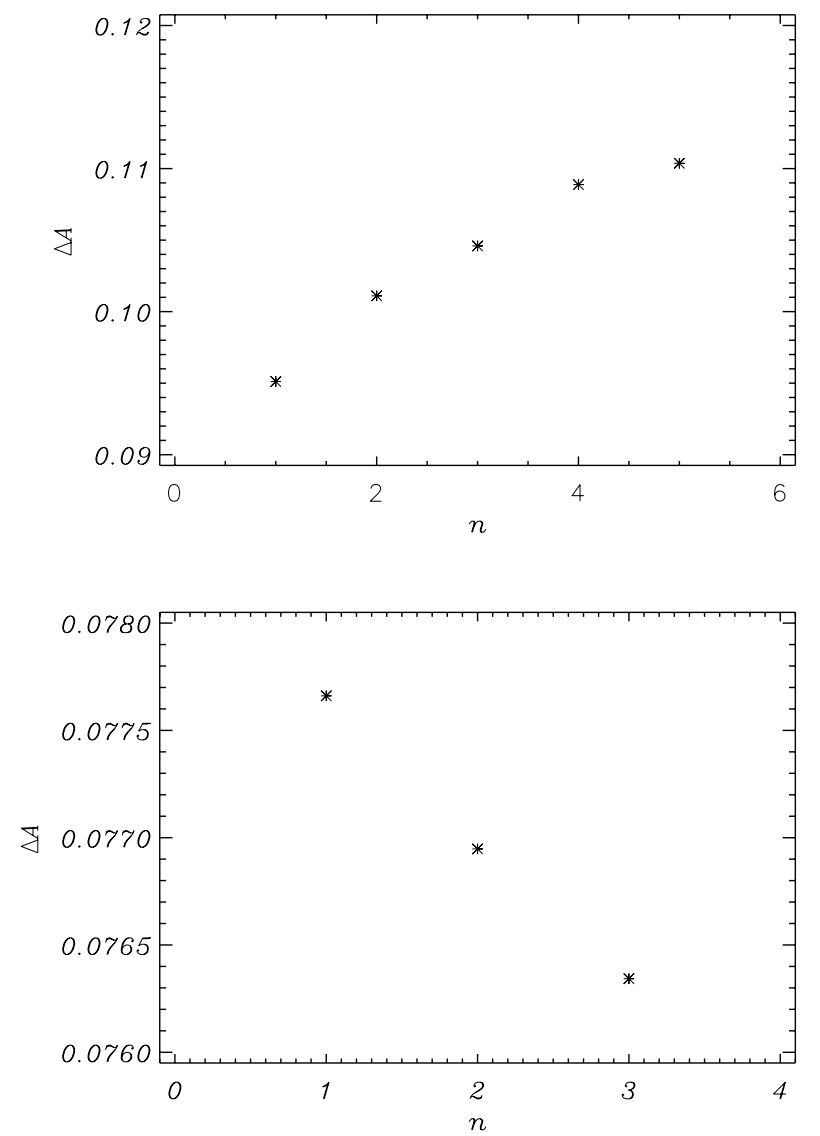

Fig. 6. Relative wave amplitude $\Delta A$ for the outer strand vs. the number of strands in the simulation region $n$ for $d=4 \mathrm{Mm}$ (top panel) and $d=10 \mathrm{Mm}$ (bottom panel). $\Delta A$ is measured in a units of the corresponding loop strand apex's height.

with $n$ for $n \geq 2$ (Fig. 7, bottom panel). We can conclude that the number of strands as well as the distance between them play, an important role in the process of wave evolution.

\section{Summary and conclusions}

The numerical simulations we performed provide insight on vertical oscillations of multi-stranded coronal loops. The results we obtained in this paper can be summarized as follows: an external pulse that is launched centrally below a loop excites a vertical kink-like mode, which bears many of the properties of the vertical oscillation observed by Wang \& Solanki (2004). We find that the wave characteristics of the numerically simulated oscillation depend significantly on a number of strands $n$, and a distance between them, $d$. In particular in the case of double-stranded loop the wave period $P$ and the attenuation time $\tau$ decline with $d$. In the case of the loop that consists of $n$ strands separated by $d=4 \mathrm{Mm}, P$ of the most outer strand grows with $n$ while $\tau / P$ essentially declines with $n$.

We conclude that the vertical oscillations of coronal loops observed by TRACE (Wang \& Solanki 2004) could be excited by propagating fast magnetosonic pulse from the flaring region below the loop. The period of the observed oscillations is determined by the geometry of the loops, as well as by the magnetic and the density structure of the loops. Based on the results of Brady \& Arber (2005), Brady et al. (2006), Verwichte et al. (2006a-c), Selwa et al. (2006a,b) and Diáz et al. (2006) we
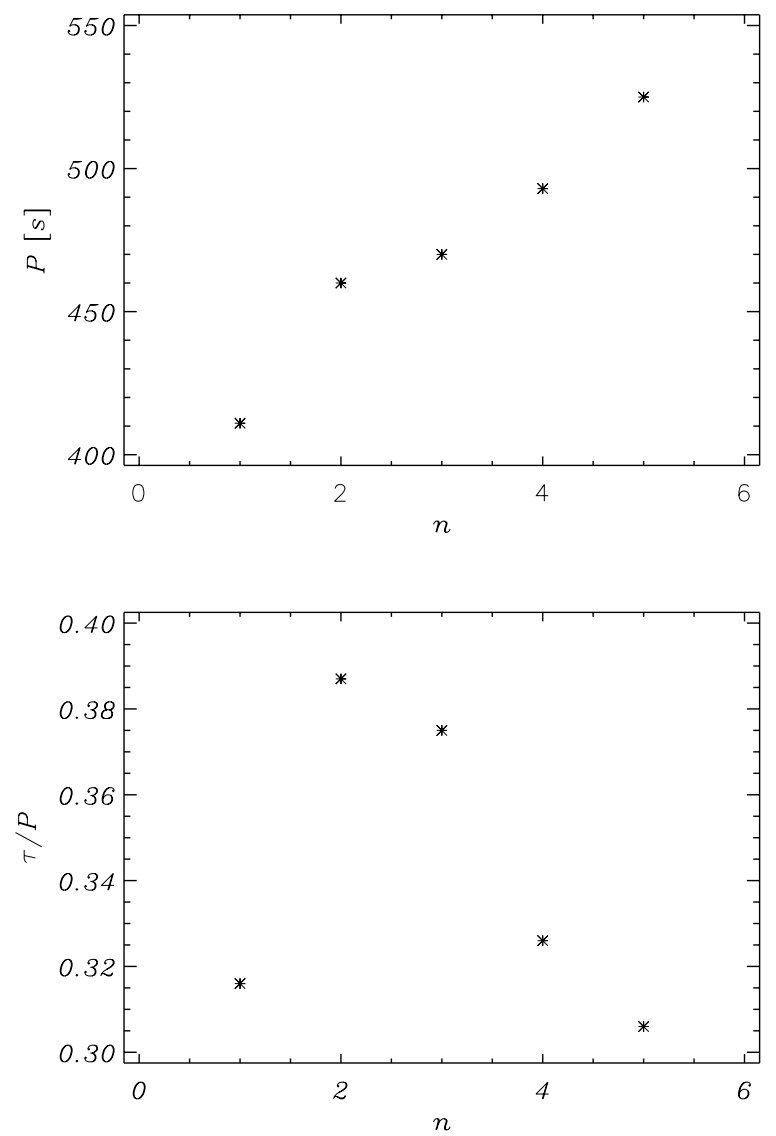

Fig. 7. Wave period $P$ (top panel) and the ratio of the attenuation time to the wave period $\tau / P$ (bottom panel), evaluated in the outer strand vs. a number of strands in the simulation region, $n$.

can conclude that the ideal damping of oscillations is due to the leakage of the fast waves from the multi-stranded loop arcade structure, while the damping time is determined by the number of strands and their mutual distance that affect the leakage rate.

In the present study we neglected gravity (Erdélyi \& Verth 2006), twisted magnetic field (Erdélyi \& Carter 2006), resistive effects, and Alfvén waves, which may play a role in the damping of multithreaded loops (e.g., Ofman \& Aschwanden 2002; Ofman 2005). Also, the coupling between the fast magnetosonic waves and Alfvén waves which takes place in a sheared arcade (Arregui et al. 2004) was neglected in this 2D study. The 3D study of curved dense loop oscillations in an idealized model of an active region is currently under way by McLaughlin and Ofman (2006). Thus, resonant absorption is not modeled in this study.

Acknowledgements. The magnetohydrodynamic code used in this study was developed at the University of Washington by Ogden S. Jones, Uri Shumlak, Scott Eberhardt, Bogdan Udrea, and provided through the sponsorship of AFOSR program. K.M.'s \& M.S.'s work was financially supported by a grant from the State Committee for Scientific Research Republic of Poland, with KBN grant No. 2 PO3D 016 25. LO would like to thanks NASA Sun-Earth Connection Theory and SR\&T programs.

\section{References}

Arregui I., Oliver R., \& Ballester J. L. 2004, A\&A, 425, 729

Aschwanden M. J., \& 2005, ApJ, 634, L193

Aschwanden M. J.,\& Nightingale R. W., 2005 ApJ, 633, 499 
Aschwanden M. J., Fletcher L., Schrijver C. J., \& Alexander D. 1999, ApJ, 520, 880

Aschwanden M. J., De Pontieu B., Schrijver C. J., \& Title, A. M., 2002, Sol. Phys., 206, 99

Brady C. S., \& Arber T. D. 2005, A\&A, 438, 733

Brady C. S., Verwichter E., \& Arber T. D. 2006, A\&A, 449, 389

Del Zanna L., Schaekens E., \& Velli M., 2005, A\&A, 431, 1095

Diáz A. J., Oliver R., \& Ballester J. L. 2005, A\&A, 440, 1167

Diáz A. J., Zaqarashvili T., \& Roberts B. 2006, A\&A, 455, 709

Edwin P. M., \& Roberts B. 1982, Sol. Phys., 76, 239

Erdélyi R., \& Carter B. K. 2006, A\&A, 455, 361

Erdélyi R., \& Verth G. 2006, A\&A, submitted

Handy B. N., Deluca E. E., \& McMullen R. A. 1998, A\&A, 30, 1269

Ionson, J. A. 1978, ApJ, 226, 650

Jones O. S., Shumlak U., \& Eberhardt D. S. 1997, J. Comput. Phys., 130, 231

McLaughlin, J.\& and Ofman, L. 2006 Proc. of the 17th SOHO Workshop, ESA $S P-617$, ESTEC, Noordwijk, in press

Murawski K. 1993, Acta Astron. 43, 161

Murawski K., Selwa M., \& Rossmanith J. A. 2005a, Solar Phys., 231, 87

Murawski K., Selwa M., \& Nocera L. 2005b, A\&A, 437, 687

Nakariakov, V. M., \& Ofman, L. 2001, A\&A, 372, L53
Nakariakov, V. M., \& Verwichte, E. 2005, Living Rev. Solar Phys., 2, 3

Nakariakov V. M., Ofman L., Deluca E. E., Roberts B., \& Davila J. M. 1999, Science, 285,862

Ofman L. 2005, Adv. Space Res. 36, 1772

Ofman, L., \& Aschwanden, M. J. 2002, ApJ, 576, L153

Oliver R., Ballester J. L., Hood A. W., \& Priest E. R. 1993, A\&A, 273, 647

Roberts B., Edwin P. M., \& Benz A. O. 1984, ApJ., 279, 857

Schrijver C. J., Aschwanden M. J., \& Title A. M. 2002, Sol. Phys., 206, 69

Schmelz J. T., Beene J. E., Nasraoui K., et al. 2003, ApJ, 599, 604

Selwa M., Murawski K., Solanki S. K., Wang T. J., \& Tóth G., 2005, A\&A, 440, 385

Selwa M., Solanki S. K., Murawski K., Wang T. J., \& Shumlak U. 2006a, A\&A, 454,653

Selwa M., Murawski K., Solanki S. K., \& Wang T. J. 2006b, A\&A, in press

Smith J. M., Roberts B., \& Oliver R. 1997, A\&A, 317, 752

Verwichte, E., Nakariakov, V. M., Ofman, L., \& DeLuca E. E. 2004, Sol. Phys., 223, 77

Verwichte E., Foullon C., \& Nakariakov V. M. 2006a, A\&A, 446, 1139

Verwichte E., Foullon C., \& Nakariakov V. M. 2006b, A\&A, 449, 769

Verwichte E., Foullon C., \& Nakariakov V. M. 2006c, A\&A, 452, 615

Wang T. J., \& Solanki S. K. 2004, A\&A, 421, L33 